\title{
Implantación de Herramientas Industriales para la Optimización del Proceso Industrial Lácteo-Fermentado
}

\author{
María Guadalupe López, Molina, PhD \\ Universidad Iberoamericana Puebla, Puebla, México \\ Rafael Trujillo Bando, M. Sc. \\ Universidad Popular Autónoma del Estado de Puebla, Puebla México \\ Bernardo Reyes Guerra, PhD \\ Instituto Tecnológico y de Estudios Superiores de Monterrey, \\ Campus Puebla, Puebla, México \\ Juan Carlos Pérez García, PhD \\ Universidad Popular Autónoma del Estado de Puebla, Puebla México
}

Doi: 10.19044/esj.2019.v15n9p408 ～URL:http://dx.doi.org/10.19044/esj.2019.v15n9p408

\section{Resumen}

Dentro de la industria láctea, existe la problemática provocada por la merma de materias primas en la fabricación de productos lácteos. En este trabajo se realiza un diagnóstico del estado actual en una planta y se establece una propuesta de implantación de herramientas industriales para mejorar la gestión del proceso. En este proyecto se utilizaron herramientas industriales basadas en manufactura esbelta y seis sigma que incluye técnicas como SMED, Jidoka, una modificación a la Metodología 5's, Poka Joke, Kanban y Kaizen. Así mismo, se hicieron ajustes al programa de mantenimiento, se utilizaron estándares visuales, procedimientos de operación estándar y pensamiento sistémico. Como resultado de este estudio se logró que los costos del tercer trimestre resultaran menores en relación a los costos del primer trimestre en promedio para los 81 productos analizados. No se rechaza ninguna de las hipótesis de investigación, ya que los elementos individuales de estudio reflejan que el costo fue menor en el tercer trimestre de estudio del 2013. Este proyecto reportó un ahorro de $0.37 \%$ entre los precios por kilo, lo que se considera bastante razonable para un mercado con margen de utilidad limitado. El enfoque de esta investigación generó un abanico de propuestas para lograr implementar mejoras al proceso industrial considerando, como áreas de oportunidad las ausencias de manufactura esbelta en diversos puntos del mismo. 
Palabras claves: Herramientas industriales, Optimización del proceso industrial, Lácteo-fermentado, Manufactura esbelta, Seis sigma

\title{
Implementation of Industrial Tools for the Optimization of the Dairy-Fermented Industrial Process
}

\author{
María Guadalupe López Molina, PhD \\ Universidad Iberoamericana Puebla, Puebla, México \\ Rafael Trujillo Bando, M. Sc. \\ Universidad Popular Autónoma del Estado de Puebla, Puebla México \\ Bernardo Reyes Guerra, PhD \\ Instituto Tecnológico y de Estudios Superiores de Monterrey, \\ Campus Puebla, Puebla, México \\ Juan Carlos Pérez García, PhD \\ Universidad Popular Autónoma del Estado de Puebla, Puebla México
}

\begin{abstract}
Within the dairy industry, there is the problem caused by the loss of raw materials in the manufacture of dairy products. In this work a diagnosis of the current state in a plant is made and a proposal for the implementation of industrial tools is established to improve the management of the process. In this project, industrial tools based on slender manufacture and six sigma were used, including techniques such as SMED, Jidoka, a modification to the Methodology 5's, Poka Joke, Kanban and Kaizen. Likewise, adjustments were made to the maintenance program, using visual standards, standard operating procedures and systemic thinking. As a result of this study, the costs of the third quarter were lower compared to the costs of the first quarter on average for the 81 products analyzed. None of the research hypotheses is rejected, since the individual elements of the study reflect that the cost was lower in the third quarter of the 2013 study. This project reported a saving of $0.37 \%$ between prices per kilo, which is considered quite reasonable for a market with limited profit margin. The focus of this research generated a range of proposals to achieve improvements to the industrial process, considering as areas of opportunity the absences of lean manufacturing in various points of the same.
\end{abstract}


Keywords: Hambol Region, Erigeron floribundus, Sclerotium rolfsii, Colletotrichum musae, Côte d'Ivoire

\section{Introduction}

La industria láctea es una de las más importantes dentro del segmento de alimentos, por año se producen cerca de 398 millones de metros cúbicos de leche a nivel mundial dentro de los cuales México ha contribuido con 9.7 millones, un $2.5 \%$ del total. Dentro de esta industria uno de los derivados con alto impacto son los productos lácteo-fermentados, conocidos popularmente como yogurt.

En la actualidad, dentro de la industria láctea, existe la problemática provocada por la generación de merma de materias primas en la fabricación de productos lácteos, además se busca la reducción de los costos de operación sin sacrificar la calidad de sus productos. En éste trabajo se realiza un diagnóstico del estado actual en una planta, se establece una propuesta de implantación de herramientas industriales para mejorar la gestión del proceso bajo un análisis puntual del flujo del sistema, y se presenta una estrategia de calidad efectiva que redujo los costos al aplicarla.

\section{Desarrollo}

La razón principal por la cual se desarrolla este estudio es concreta: lograr establecer una propuesta basada en herramientas cuantitativas que genere un valor agregado al proceso actual de una empresa dedicada a la elaboración de productos lácteos fermentados. Teniendo como puntos de anclaje: los procedimientos de arranque y paros de línea, ejecución de programas de mantenimiento, aplicación de cultura esbelta y seis sigma, con las cuales se mantendrá claro el objetivo de poder alcanzar un incremento en la productividad, una reducción en los costos operacionales con un enfoque en la cultura organizacional y la visión del negocio.

Se pueden encontrar revisiones en la literatura del progreso de la investigación en la aplicación de herramientas industriales -como manufactura esbelta y seis sigma- durante la última década (Dhiravidamani, et al. 2018; Baby, Prasanth \& Jebadurai, 2018; Nallusamy, \& Ahamed, 2017; Rymaszewska, 2017; Zhou, 2016; Al-Ashaab et al, 2016; de Lima et al, 2016; de Medeiros et al, 2016; Jasti, \& Kodali, 2016; Shah \& Hussain, 2016; Bortolotti, Boscari, \& Danese, 2015; Grudowski, Wiśniewska \& Leseure, 2015; Pirasteh \& Kannappan, 2013; Smith, 2013; Akbulut-Bailey, Motwani, \& Smedley, 2012; Timans et al, 2012).

La variables de entrada (independientes) en este estudio son: la variación de uso en las recetas de fabricación desde base cero, el reproceso generado en semielaborados así como en producto terminado y el sobredosificado que se agrega a cada pieza producida adicional a lo esperado. La 
variable de respuesta (dependiente) es el desarrollo exitoso de la manufactura esbelta operacionalizada como un beneficio en la variación del costo de materias primas en producción, comparando el primer y tercer trimestre del 2013.

\section{Seis Sigma}

Es una metodología cuya finalidad es la reducción de variaciones en proceso, que tuvo sus orígenes en Motorola en los años ochenta. Las variaciones se refieren a la desviación estándar, representada por la letra griega sigma.

La metodología Seis Sigma se basa en las siguientes premisas: mejora por proyectos, cero o mínima inversión de capital, proyectos de rápida implementación (6 a 8 meses) y proceso disciplinado con decisiones basadas en datos.

Esta metodología utiliza herramientas y métodos estadísticos para definir problemas, medición para obtener la información, análisis de la información recolectada, incorporación de acciones en los procesos y finalmente, el control o rediseño de los mismos, con la finalidad de alcanzar etapas ideales, lo que a su vez genera un ciclo de optimización consistente en Definición, Medición, Análisis, Implementación y Control, denominado DMAIC, por sus siglas.

\section{Manufactura Esbelta}

La manufactura esbelta utiliza varias herramientas que ayudan a eliminar todas las operaciones que no le agregan valor al producto, servicio y a los procesos, aumentando el valor de cada actividad realizada y eliminando lo que no se requiere; reduce desperdicios y mejora las operaciones, basándose siempre en el respeto al trabajador.

Los desperdicios que se pretende eliminar son los siguientes siete: sobreproducción, que consiste en hacer más de lo que el cliente ha solicitado; inventario, referente a tener más producto a la mano del que el cliente necesita; transportación, lo que sucede al mover el producto más de lo que es necesario; sobre procesamiento, consiste en hacer más cosas al producto de las que el cliente pidió; espera, que se refiere a cualquier momento en el que el valor no puede ser agregado por causa del retraso; movimiento, cualquier desplazamiento extra del operador cuando está realizando una secuencia de trabajo y corrección consiste en cualquier cosa no hecha bien desde la primera vez, que requiera re-trabajo o inspección.

Sin importar la rama o tipo de industria que desee aplicarlo, el rol de los líderes en la implementación de la manufactura esbelta es intercambiar su conocimiento (acerca de lo que funciona y lo que no funciona), ser abierto a 
cambiar aquello que no marcha correctamente e intentar cosas nuevas aun cuando sepan que no funcionarán, luego buscar cómo hacer que funcionen.

Las características de la manufactura esbelta son que define el valor e identifica la cadena de valor para su producto, elimina todos los pasos innecesarios en toda cadena de valor y crea flujo de valor: que todo el proceso fluya suave y directamente de un paso que agregue valor a otro, desde la materia prima hasta el consumidor. Una vez diseñado el flujo, serán capaces de producir en base a órdenes de los clientes en vez de hacerlo en base a pronósticos de ventas a largo plazo.

Algunas de las herramientas que utiliza la manufactura esbelta y sus características utilizadas en fábrica para la elaboración de este trabajo son:

Kaizen. Se basa en el mejoramiento continuo, que se sustenta en dos pilares: equipos de trabajo e ingeniería industrial; los cuales se utilizan para optimizar los procesos productivos. El método se enfoca a la gente y a la estandarización de los procesos, requiriendo de un equipo integrado por personal de producción, mantenimiento, calidad, ingeniería, compras y otros empleados que se considere pertinente. También se enfoca a la eliminación de desperdicio en cualquiera de sus formas.

Metodología 5s. Está incluida dentro de lo que se conoce como Kaizen. En esencia se refiere a la creación y mantenimiento de áreas de trabajo más limpias, organizadas y seguras; es un cambio realizado por la gente para la gente. Las 5s provienen de términos japoneses: seiri (organizar), seiton (clasificar), seiso (limpiar), seiketsu (estandarizar), shitsuke (disciplina).

SMED (Single Minute Exchange of Die). Su objetivo es incrementar la flexibilidad al estar disponible para reaccionar rápidamente a necesidades clave de los clientes reduciendo inventarios. Algunos de los beneficios que tiene esta herramienta son producir en lotes pequeños, reducir inventarios, procesar productos de alta calidad, reducir los costos, tiempos de entrega más cortos para ser más competitivos, tiempos de cambio más confiables.

Justo a tiempo. Es una filosofía industrial cuya idea básica es producir un artículo en el momento que es requerido para que éste sea vendido o utilizado por la siguiente estación de trabajo en un proceso de manufactura. Dentro de la línea de producción se controlan en forma estricta no sólo los niveles totales de almacenamiento, sino también el nivel de inventario entre las células de trabajo.

Pull system. En este sistema las referencias de producción provienen del centro de trabajo precedente. Cada estación de trabajo dispone de la cantidad 
exacta para sacar las partes disponibles a ensamblar o agregar al producto en la siguiente estación, esto corresponde a comenzar desde el final de la cadena de ensamble e ir hacia atrás hacia todos los componentes de la cadena productiva, incluyendo proveedores y vendedores. Este sistema permite reducir inventario y maximiza la retroalimentación.

Estándares visuales. Un control visual es un estándar representado mediante un elemento gráfico o físico, de color o numérico muy fácil de ver, la estandarización se transforma en gráficos y éstos se convierten en controles visuales. Cuando se logra implementar correctamente, sólo hay un sitio para cada cosa y podemos decir de modo inmediato si una operación particular está procediendo normal o anormalmente.

Kanban. Es una herramienta de manejo de flujo de materiales en una línea de ensamble, como una etiqueta de instrucción que contiene información que sirve como orden de trabajo, se vuelve un dispositivo de dirección automático que da información acerca de lo que se va a producir, su cantidad, sus medios y su transporte. El objetivo principal de esta herramienta es el control de la producción y la mejora de los procesos.

Jidoka. Permite detectar y corregir defectos de producción utilizando mecanismos y procedimientos que detectan una anomalía en el sistema, llegando al punto de detener una línea de producción para evitar la elaboración de productos defectuosos. Sus características son aseguramiento de la calidad el $100 \%$ del tiempo, prevención de averías de equipos, uso eficaz de la mano de obra, verificación más eficiente de los defectos de calidad, canal de comunicación directo e integración hacia adelante de la mejora específica.

Poka Joke. Ayuda a prevenir los errores antes de que sucedan, o los hace obvios para que el trabajador los corrija a tiempo. Sus características principales son procesos simples y baratos, ya que, si fueran demasiado complicados o caros, su uso no sería rentable, se lleva a cabo $100 \%$ de la inspección, en el lugar donde ocurre el error, para proporcionar retroalimentación rápidamente y que los errores puedan corregirse.

Procedimientos de Operación Estándar (SOP, por sus siglas en inglés). Son métodos prescritos o establecidos para que sean seguidos como rutina en la aplicación de operaciones o tareas específicas. Deben considerar el objetivo y frecuencia para realizar las actividades, la persona que será encargada de realizar la tarea, una descripción del procedimiento que se quiere aplicar incluyendo sus etapas y las acciones correctivas que deben llevarse a cabo si es necesario. 


\section{Metodología}

La variables de entrada (independientes) en este estudio son: la variación de uso en las recetas de fabricación desde base cero, el reproceso generado en semielaborados así como en producto terminado y el sobredosificado que se agrega a cada pieza producida adicional a lo esperado; la variable de respuesta (dependiente) es el desarrollo exitoso de la manufactura esbelta operacionalizada como un beneficio en la variación del costo de materias primas en producción, comparando el primer y tercer trimestre del 2013.

Para la aplicación de esta propuesta se estableció lo siguiente como línea de método:

- Involucrar a la gerencia en la visión de implementar procesos esbeltos.

- Formar un comité de mejora con especialistas del área productiva, identificar el proyecto líder y plantear objetivos.

- Generar un plan estratégico integral que encuadre los requerimientos que cubran y den solución al planteamiento del problema inicial.

- Comunicar el plan y la visión a toda la organización a través de publicación de objetivos por alcanzar y tiempos de implementación.

- Formar un equipo de trabajo multidisciplinario, quienes serán los líderes del pensamiento esbelto en las áreas donde se ejecuten las iniciativas.

- Entrenar teórica y prácticamente a los líderes en las herramientas a implementar y realizar benchmarking con otras plantas.

- Seleccionar una línea piloto e implementar las herramientas durante el primer mes para después replicar en las demás líneas de producción.

- Implementar en el resto de las áreas.

- Trabajar durante el segundo mes replicando herramientas en las demás maquinarias, documentando prácticas exitosas.

- Evaluar los resultados y obtener retroalimentación.

- Entrar en la etapa de control, delegando a los supervisores el rol de empoderamiento y apoyando a la línea a que sea auto-dirigida por los operadores directos

- Una vez que se esté satisfecho con los resultados, seleccionar otra herramienta, la que mejores resultados genere a la organización e implementarla.

\section{Hipótesis}

$\mathrm{Ha}_{1}$ : Existe correlación de los costos por kilo producidos en el tercer trimestre del año 2013 contra los del primer trimestre del mismo año por causas de reproceso. 
$\mathrm{Ha}_{2}$ : Existe correlación de los costos por kilo producidos en el tercer trimestre del año 2013 contra los del primer trimestre del mismo año por causas de receta base cero.

$\mathrm{Ha}_{3}$ : Existe correlación de los costos por kilo producidos en el tercer trimestre del año 2013 contra los del primer trimestre del mismo año por causas de sobredosificación.

\section{Medición}

Dentro de la metodología propuesta para este caso de estudio se considera la estandarización, evaluación de resultados y retroalimentación, lo cual ayuda a la implementación industrial.

En general es una metodología que abarca partes cardinales dentro de la implementación en donde se planea estratégicamente tener cuatro etapas fundamentales para un desarrollo adecuado y arranques verticales, estas etapas son descritas a continuación:

Decisión del cambio. Es necesario convencer a los gerentes de producción de la importancia el cambio si se quiere tener éxito en la implementación, para esto se planea mostrar la evolución estadística del 2012 en las líneas de producción. Una vez demostrada la necesidad de instaurar el proceso de mejora continua, los pasos siguientes son demostrar las características y cualidades del plan estratégico, mostrando además las diferencias de este en relación a otros sistemas de calidad.

Despliegue de objetivos. Como parte de esta etapa se establecen los sistemas de información para publicar los alcanzables y trazar un ideal para el equipo de trabajo, de tal manera que se busque motivar a los colaboradores a sumarse a las iniciativas de reducción de mermas. Se forman los primeros grupos de trabajo en función del plan estratégico alineado con los beneficios esperados para la empresa.

Desarrollo del proyecto. Dentro de esta etapa es primordial definir los requerimientos de los clientes externos e internos y la forma en que se medirán los logros de dichas especificaciones. Sólo entonces, el equipo de trabajo procederá a aplicar la metodología descrita anteriormente, de tal manera que se implementen efectivamente las iniciativas de mejora fundamentadas en técnicas de manufactura esbelta.

Evaluación de beneficios. En esta última etapa se determinan los resultados producidos luego de la implementación de los cambios resultantes del desarrollo de las diversas iniciativas correspondientes al plan estratégico. Estos resultados se manifiestan tanto en niveles de rendimientos, volumen de 
generación de desperdicio, costos por kilo producido, ahorros obtenidos por ejecución y cambio del modelo de pensamiento en los operadores siendo esto necesario para el sostenimiento del proyecto.

\section{Discusión y Análisis de Resultados}

Los resultados se reflejaron al corto plazo de la implementación de las herramientas para gestión industrial, de esta manera se establece una cadena de resultados alineados a lo encontrado en la fábrica.

En este caso se detectó que las materias primas y los embalajes representan en conjunto el $70 \%$ del costo, por tanto, es de suma importancia para la optimización de los recursos tener una gestión cuidando estos dos rubros. Se observó que las causas que más afectan la generación de merma son aquellas relacionadas con: el método en un $35 \%$, el hombre en un $26 \%$ y las máquinas en un $25 \%$, siendo éstas las causas contribuyentes en un $86 \%$ a la generación de merma y por ende foco de atención para resolver.

Se realizó un análisis de distribución, en donde se detecta que las causas de reproceso son la actividad principal a erradicar, para obtener resultados exitosos se debe lograr enfocar los esfuerzos en la línea que tiene mayor potencial de mejora y así consolidar los resultados en el menor tiempo posible.

Posteriormente se presenta la necesidad de realizar una regresión para entender si estas tres variables, detectadas mediante estrategias administrativas de manufactura esbelta y análisis estadístico de la información, tienen una relación directa con la optimización del proceso visto desde el punto de vista económico en el precio por kilo producido, teniendo como soporte una gestión estratégica en los estándares operativos de la fábrica y funcionando los resultados arrojados como una herramienta de análisis para la toma de decisiones fundamentada en métodos numéricos.

Se analizan los productos que conforman la cartera de producción de la fábrica modelo en cuestión durante el primer trimestre del 2013, su volumen de producción generado, la variación del costo de materia prima en pesos (por ejemplo, presupuesto de 30 pesos $/ \mathrm{kg}$ en materia prima, costo real materia prima 32 pesos $/ \mathrm{kg}$, variación 2 pesos), el porcentaje de pérdida en materia prima por reproceso, por base cero y por sobredosificación.

Se realiza el estudio de regresión, teniendo como mejor modelo de ajuste el lineal. Se tiene que los resultados obtenidos sustentan la selección de las variables elegidas, el coeficiente de correlación múltiple de Pearson es 0.99 con lo cual se concluye que la correlación de estas variables independientes con la dependiente es alta, lo que se demuestra estadísticamente. De igual manera se refleja en el coeficiente de determinación que es de 0.98 . Por tanto, el 98\% de las variables dependientes le dan razón de ser a la independiente.

Los resultados de las curvas ajustadas de regresión (figuras 1 a 3) con las cuales se establece una dispersión de los valores reales y el pronóstico 
generado en donde se aprecia de la misma condición la linealidad de las variables, la primera de ellas es la gráfica de regresión relacionada al reproceso.

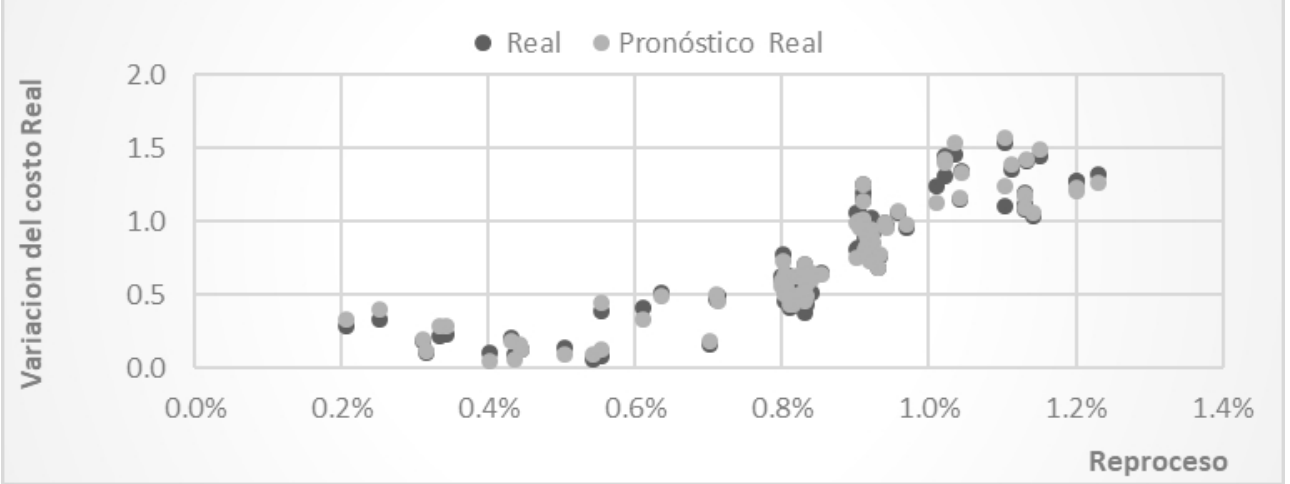

Figura 1.Curva de Regresión Ajustada Reproceso. Fuente: Creación propia

Los datos presentan una tendencia positiva creciente en relación al incremento del costo real, correlacionándose con un coeficiente de 0.868 .

El comportamiento bajo el mismo escenario de análisis, pero con la relación de regresión ajustada para la variable receta base cero:

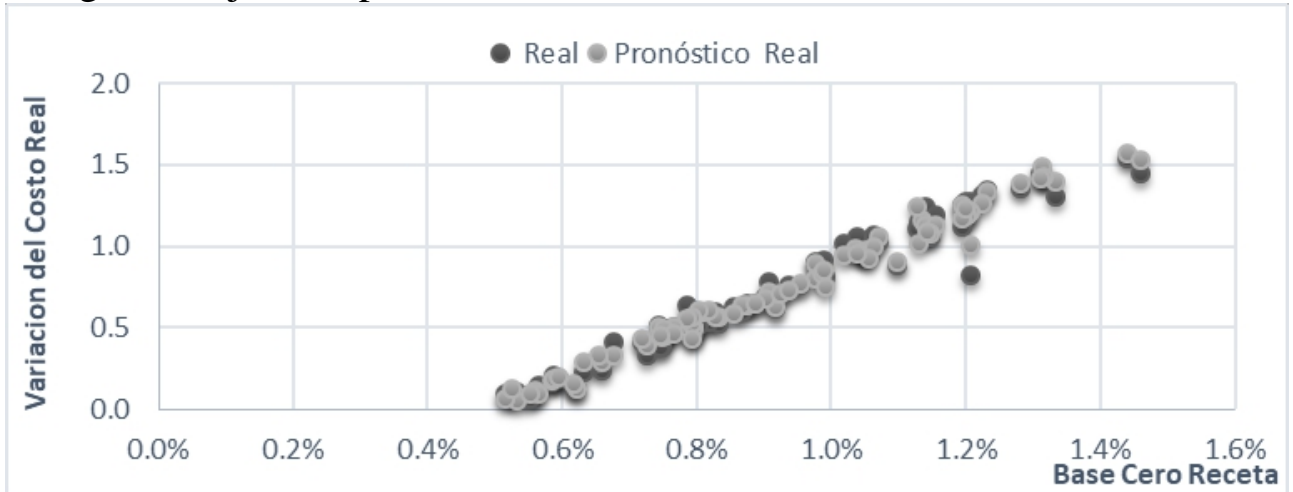

Figura 2. Curva de Regresión Ajustada Base Cero Receta. Fuente: Creación propia

En este caso se muestra una linealidad con un coeficiente de correlación de 0.99 .

Finalmente, la tercera y última variable considerada para este estudio, sobredosificación de producto. 


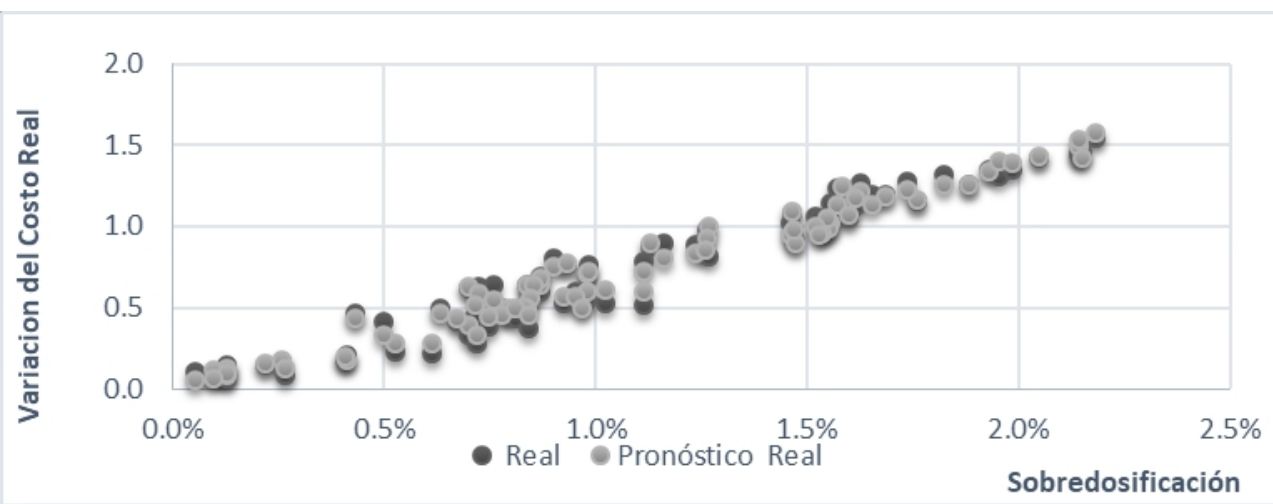

Figura 3. Curva De Regresión Ajustada Sobredosificación. Fuente: Creación propia

Resultados arrojados por la regresión muestran un coeficiente de correlación de 0.982 .

Habiendo identificado la distribución del gasto industrial, distribución ponderada de uso por línea de producción, bloques principales de generación de merma, las líneas de impacto donde se debe enfocar los esfuerzos, la variación del costo que presenta cada producto, los factores de merma bajo una trazabilidad porcentual, la regresión de correlación lineal entre la variable dependiente y las independientes, es necesario saber por dónde comenzar teniendo un panorama de soporte para establecer las estrategias de calidad y poseer un mapa de procesos que nos muestre una visión panorámica sobre las áreas de oportunidad.

En relación a ello se detectaron, gracias a los análisis cuantitativos realizados para la toma de decisiones por parte del equipo de trabajo involucrado, las estrategias de calidad que serían aplicadas dada la condicionalidad de cada causa de generación de merma y se determinaron las siguientes como las actividades estandarte para cada iniciativa, no con ello actuaron de manera exclusiva, se han implementado las mismas del mismo modo global a nivel fábrica sin embargo se hizo un mayor énfasis en su aplicación y se pretende obtener un resultado final optimo, las actividades mencionadas se muestran en la siguiente tabla:

Tabla 1. Estrategia de Calidad por Variable Independiente.

\begin{tabular}{l|l|l}
\hline Reproceso & Base cero & Sobredosificado \\
\hline SMED & Pensamiento sistémico & Kaisen \\
\hline Programa de mantenimiento & Standard Operation Procedures & Estándares Visuales \\
\hline Jidoka & Poka Joke & Revisión dosificadoras \\
\hline Aplicación de 3 s & Kanban & \\
\hline
\end{tabular}

Fuente: Creación propia 
A continuación, se detalla cada una de las estrategias implementadas así como los resultados obtenidos durante el tercer trimestre del 2013 gracias a su implementación, en la siguiente parte del reporte de investigación se muestran las principales aportaciones positivas por la implementación de las estrategias de calidad planeadas y los resultados más significativos reflejados.

Se muestran a detalle cuales fueron los impactos en relación al primer trimestre y de este género su beneficio operacional y económico de haber tomado la decisión de incluirlos en el plan maestro de operaciones para el ciclo 2013.

Reproceso. Las condiciones de reproceso se optimizaron debido a las siguientes iniciativas de aplicación industrial, las acciones que se tomaron dieron estructura a las actividades de reproceso y buscan reducir el impacto en el costo por fallas en proceso, las siguientes herramientas se detallan en los siguientes apartados.

SMED. Gracias a la implementación de la metodología SMED en las líneas de producción, se logró una reducción del 20\% en tiempos de limpieza para todas las máquinas, ampliando así la utilización por turno y optimizando los arranques y paros de línea, siendo más verticales en la puesta en marcha para volver a iniciar ciclo de operación continua y generando menos merma por una de las causas principales de generación, el arranque de línea, pasando así de 57.9 toneladas de mema en el primer trimestre por 38.6 toneladas para el tercer trimestre del 2013, lo que representa un beneficio significativo del $34.6 \%$ incluso en condiciones donde la producción se incrementó en volumen.

Programa de mantenimiento. Se revisaron los planes de mantenimiento, los cuales se encontraban organizados de manera aislada acorde a las necesidades de la máquina exclusivamente, no se tenía una consideración de dos conceptos clave, el primero de ellos fue que los tiempos netos de producción se veían afectados ya que el número de intervenciones por los diferentes departamentos no estaban siendo coordinados, siendo la primera tarea lograr empatar los ajustes de máquina con lo requerido por logística, muestreos de calidad, pruebas de innovación, logrando así una utilización funcional de las muestras tomadas de la línea.

En segundo plano se reestructuró el modelo de mantenimiento para dar un mayor monitoreo al comportamiento del codificador y se instaló un depósito de tinta con mayor capacidad, de tal forma se asegurará la producción continua por esta causa de falla, los resultados mostrados por reproceso debido a mal codificado se redujeron durante el periodo de ejecución de 31.5 a 24.4 toneladas, un beneficio del $22.4 \%$. 
Se atacaron las causas referentes a mal sellado, el plan de mantenimiento fue modificado para dar una revisión a mayor detalle de la presión de los mástiles de sellado y la temperatura a la que son aplicadas las tapas de las botellas, logrando estandarizar la temperatura de sellado en $170^{\circ} \mathrm{C}$ y mostrando un beneficio al pasar de 14.9 a 13.2 Toneladas de reproceso relacionadas a este problema, un beneficio del $11.3 \%$

Jidoka. Se aplicó esta metodología de manufactura esbelta con el fin de verificar directamente en la línea del proceso problemas relacionados con formación de partículas grumosas, que son entendidos a nivel industrial alimenticio como grumos formados por falta de una homogenización de los ingredientes. La aplicación de Jidoka funcionó para comunicar rápidamente al departamento de fabricación que el producto necesitaba un ciclo adicional de agitación para reducir los lotes afectados por esta condición, esto representó una reducción de 56.1 a 32.6 toneladas durante el primero y tercer trimestre del 2013 respectivamente, un $41.7 \%$ de beneficio registrado.

Metodología 3s. Normalmente esta metodología es conocida como $5 \mathrm{~s}$ relacionado con orden y limpieza, sin embargo, se consideró para la realización de esta estrategia comenzar una campaña con las primeras tres de ellas de tal forma que se consolidaran por etapas y fueran sostenibles en el largo plazo.

Se realizó una evaluación inicial de las áreas productivas y se encontró que el porcentaje de orden y limpieza era $70.1 \%$ lo cual no podía ser aceptable para una empresa dedicada al giro alimenticio, con lo cual se logró establecer un resultado en esta metodología superior al $80 \%$ de calificación, este objetivo fue superado con un $83.9 \%$ de calificación total planta, un excelente resultado gracias a la implementación de la campaña "Unidos! 3s" que fue bien adoptada por el personal operativo, y reducir de 22.9 a 16.1 toneladas un $30.2 \%$ por las causas de cambios de sabor, plásticos equivocados y falta de material en línea, ésta metodología sumó esfuerzos con el departamento de calidad en la reducción de contaminación por cuerpos extraños dentro del producto, reduciendo los reclamos del consumidor a fábrica.

Base cero. La receta base cero es clave para beneficiar a todo el proceso, las metodologías aplicadas fueron relevantes debido al volumen de manejo que impacta esta condición y se reportan casos de éxito que se detallan más delante de este trabajo.

Pensamiento sistémico. Se capacitó al personal sobre las herramientas de pensamiento sistémico y como lograr entender las necesidades del sistema de manera integral. Posteriormente se crearon equipos multidisciplinarios de 
trabajo para encontrar posibles soluciones a los problemas declarados en la fábrica y relacionados con la perdida por variaciones en base cero.

En este sentido las actividades que se realizaron implicaron un beneficio en donde se incrementó la capacidad 5\% en la base pasteurizada por la habilitación de dos tanques que tenían averías y se mandaron a recuperar para este proyecto, gracias a esta aplicación de pensamiento sistémico se logró incrementar la vida de utilización de las bases lácteas de 48 a 56 horas ampliando la utilización por ciclo de fabricación de 70 a 80 Toneladas de llenado continuo en línea.

Adicionalmente como resultado del incremento en la etapa de enfriamiento de 7,200 a 10,000 Kg/Hr se logró estabilizar de manera clara la fermentación de los componentes de la formula y se redujeron las condiciones de reproceso en el periodo comparativo trimestral en el caso de producto líquido de 59.1 a 35.5 toneladas un beneficio del $40.1 \%$, y en cuanto a desviación de Color de 32.3 a 29.7 toneladas un beneficio de $8.1 \%$

Procedimientos operación estándar. Esta fue una de las actividades que lograron consolidar con un nivel de organización interesante que se vio reflejado en el $13.38 \%$ de reducción en la causa de desviación de producto con espuma, pasando de 17.4 a 15.1 toneladas de reproceso por esta causa. La estandarización de los procedimientos de operación fue un trabajo que implicó investigar las variables independientes que afectaban al proceso, la más representativa fue detectar que la velocidad de llenado no estaba estandarizada, lo realizado aquí fue establecer los parámetros de velocidad para el llenado dependiendo del vaciado del tanque y su condición mientras se vierte en el proceso de llenado.

Poka Joke. Se implementó la metodología esbelta Poka Joke, un reflejo de ello fue la calibración aguda de presión del aire comprimido en el equipo de corte para las tapas de botella, de esta forma se solucionó el problema de distribución de estos elementos y se redujeron las fallas por producto sin tapa de 10.8 a 9.9 toneladas un beneficio del $15.2 \%$ durante el periodo.

Kanban. Se aplicó esta metodología y se implementó un sistema de tarjetas de tal manera que se alinearon los requerimientos del mercado, las condiciones de llenado y embalaje para alinear las bases con mayor precisión al momento de su entrega, con lo cual la base láctea permanecía menos tiempo en espera una vez preparada y se dosificaba en un menor tiempo, ayudando esto a no continuar fermentando en los tanques de fabricación y reduciendo las condiciones para que se acidificara el producto, esto mostró un beneficio tangible al reducir los desvíos por producto ácido de 29.3 a 24.3 toneladas, un beneficio del $16.8 \%$ dentro del periodo de estudio. 
Sobredosificación. La sobredosificación es un tema complejo de medir por la velocidad y el volumen de llenado, pero se implementaron acciones a las condiciones básicas para obtener resultados en operación, la aplicación de métodos de ingeniería industria fue quien aportó las condiciones necesarias para optimizar la situación encontrada.

Kaizen. Con esta herramienta se detectó un problema que afectaba directamente la sobredosificación debido a las variaciones internas del diámetro de la manguera, donde se mantenía una presión positiva y al reducir el área transversal de la manguera el flujo circulaba a mayor velocidad, afectando así el gramaje por botella que se dosificaba en la maquinaria, la condición fue detectada y solucionada con lo cual se aportó a la reducción de este indicador durante el periodo de evaluación.

Estándares visuales. Se implementaron estándares visuales para identificar claramente cuál es la manera correcta de revisar la presión de paso de los dosificadores y ser cuidadosos con los márgenes de tolerancia, de tal modo que se enfoquen en ajustar agudamente éstos dispositivos y no generar un porcentaje de dosificación fuera de parámetros, la reducción de dosificado se modificó de $1.1 \%$ excedente en el total de botellas a $0.8 \%$ con lo cual se dio un ahorro dentro del marco de necesidades y planteamiento del problema.

\section{Resultados: Comparativo Periodos}

Los resultados que se presentaron en este estudio generaron propuestas de trabajo para posteriormente implementarlas y observar que los resultados fueron un éxito en el primer plano, para llegar a generar ahorros significativos por parte de la empresa, permitiendo que el proceso se pueda llegar a gestionar de manera dinámica y en beneficio de la optimización del mismo.

La elaboración de esta herramienta de análisis nos permite a continuación generar un comparativo de resultados en base a las condiciones más significativas del modelo entre el tercer trimestre del 2013 como periodo evaluado contra la referencia previamente establecida como objetivo en el primer trimestre del 2013. Las siguientes condiciones, que se muestran en la tabla 2, son una muestra del resultado de un gran esfuerzo por parte del equipo de trabajo sobre el proceso y se refleja cómo éstas generaron un impacto positivo en la optimización industrial. 
Tabla 2. Comparativo de Periodos Q3 vs Q1

\begin{tabular}{|c|c|c|c|c|c|}
\hline $\begin{array}{c}\text { Causa } \\
\text { Atacada }\end{array}$ & Estrategia & $\begin{array}{c}\text { Problema } \\
\text { Atacado }\end{array}$ & $\begin{array}{c}\text { Primer } \\
\text { Trimestre } \\
13 \text { (Q1) }\end{array}$ & $\begin{array}{c}\text { Tercer } \\
\text { Trimestre } \\
13(\mathbf{Q})\end{array}$ & $\begin{array}{c}\text { Var.Q3 } \\
\text { vs Q1 }\end{array}$ \\
\hline \multirow{5}{*}{ REPROCESO } & SMED & $\begin{array}{l}\text { Arranque de } \\
\text { Línea }\end{array}$ & 57.9 Ton & 38.6 Ton & $-34.6 \%$ \\
\hline & \multirow{2}{*}{$\begin{array}{c}\text { Programa } \\
\text { mantenimiento }\end{array}$} & $\begin{array}{c}\text { Mal } \\
\text { Codificado }\end{array}$ & 31.5 Ton & 24.4 Ton & $-22.4 \%$ \\
\hline & & Mal Sellado & 14.9 Ton & 13.2 Ton & $-11.3 \%$ \\
\hline & Jidoka & $\begin{array}{l}\text { Producto } \\
\text { PIP's }\end{array}$ & 56.1 Ton & 32.6 Ton & $-41.7 \%$ \\
\hline & $5 \mathrm{~s}$ & $\begin{array}{l}\text { Cambio de } \\
\text { Sabor }\end{array}$ & 22.9 Ton & 16.1 Ton & $-30.2 \%$ \\
\hline \multirow{5}{*}{$\begin{array}{c}\text { RECETA } \\
\text { BASE CERO }\end{array}$} & \multirow{2}{*}{$\begin{array}{l}\text { Pensamiento } \\
\text { Sistémico }\end{array}$} & $\begin{array}{l}\text { Producto } \\
\text { Liquido }\end{array}$ & 59.1 Ton & 35.5 Ton & $-40.1 \%$ \\
\hline & & $\begin{array}{c}\text { Desviación de } \\
\text { color }\end{array}$ & 32.3 Ton & 29.7 Ton & $-8.1 \%$ \\
\hline & $\mathrm{SOP}^{\prime} \mathrm{s}$ & $\begin{array}{l}\text { Producto } \\
\text { c/Espuma }\end{array}$ & 17.4 Ton & 15.1 Ton & $-13.4 \%$ \\
\hline & Poka Joke & $\begin{array}{l}\text { Producto } \\
\text { s/Tapa }\end{array}$ & 10.8 Ton & 9.9 Ton & $-15-2 \%$ \\
\hline & Kanban & $\begin{array}{l}\text { Producto } \\
\text { Acido }\end{array}$ & 29.3 Ton & 24.3 Ton & $-16.8 \%$ \\
\hline \multirow{2}{*}{$\begin{array}{c}\text { SOBRE } \\
\text { DOSIFICADO }\end{array}$} & Kaizen & $\begin{array}{c}\text { Mangueras } \\
\text { Tapadas }\end{array}$ & 4/Ciclo & 3/Ciclo & $-25.0 \%$ \\
\hline & $\begin{array}{l}\text { Estándar } \\
\text { Visual }\end{array}$ & $\begin{array}{l}\text { Calibración } \\
\text { dosificador }\end{array}$ & $1.1 \%$ & $0.8 \%$ & $-27-2 \%$ \\
\hline
\end{tabular}

Gracias a la detección cuantitativa por medio de las herramientas estadísticas para la toma de decisiones y los instrumentales de control utilizados durante la gestión propuesta del modelo dinámico, se aprecia como los beneficios logrados fueron sólidos y superaron lo estimado al principio de la investigación.

Causas de reproceso. Gracias a las iniciativas propuestas y medidas de la investigación se logró obtener un resultado positivo reduciendo 0.16 pesos por kilo producido, un $0.87 \%$ de beneficio lo cual es prácticamente ideal frente al $0.90 \%$ que se optimizó la generación de mermas gracias a este modelo de gestión dinámica, sin embargo, existieron irreversibilidades del sistema y sobre gastos por condiciones externas a esta investigación por energía, embalaje y mano de obra directa que opacaron el resultado operativo en el costo por kilo trimestral. 
Beneficio económico. Este proyecto reportó un ahorro de $0.37 \%$ entre los precios por kilo, pasando de 18.81 a 18.74 en costo, teniendo un potencial integrado de 24.8 Millones al año, sin embargo, después de considerar las irreversibilidades presentadas por energía, embalaje y mano de obra se reportaron ahorros por 10.8 millones de pesos calculados en un horizonte anual, sin aportación de capital adicional a la implementación de iniciativas. Estas iniciativas funcionaron como motor de apoyo al plan de ahorro de la fábrica y sus costos de operación en el ejercicio financiero 2013.

\section{Comprobación de Hipótesis}

Habiendo estudiado los efectos de manera global, es significativo comprobar los hechos del estatus quo contra lo deseado inicialmente para analizar numéricamente que este efecto mostrado es correcto para toda la población de productos, la hipótesis nula indica que los costos del tercer trimestre (Q3) serán mayores o iguales que los presentados en el primer trimestre $(\mathrm{Q} 1)$, caso contrario para la hipótesis de investigación donde el primer trimestre $(\mathrm{Q} 1)$ es mayor que el tercer trimestre $(\mathrm{Q} 3)$ debido a la implementación de herramientas de manufactura esbelta.

Se muestran en el siguiente análisis los valores en donde no se rechazan ninguna de las hipótesis de investigación, ya que los elementos individuales de estudio reflejan que el costo fue menor en el tercer trimestre de estudio del 2013.

Se obtiene un p-value por cada hipótesis por debajo del 5\% de significancia, concluyendo así lo anteriormente mencionado.

De esta prueba se reconoce como válidos, bajo el marco de investigación definido en este estudio, que los costos del Q3 resultaron menores en relación a los costos del Q1 en promedio para los productos analizados, mostrando un costo por kilo inferior en 0.0867 pesos, una diferencia bastante razonable para un mercado con margen de utilidad limitado.

\section{Conclusión}

El enfoque de esta investigación generó un abanico de propuestas para lograr implementar mejoras al proceso industrial considerando como áreas de oportunidad las ausencias de manufactura esbelta en diversos puntos del mismo. Adicionalmente el fundamentarlo en un modelo de pensamiento sistémico que sea flexible de implementar y ayude a la empresa a solidificar las bases del comportamiento humano para crear conciencia sobre su gestión de generación de mermas, manteniendo en el largo plazo un escenario sostenible para la operación futura y se establezca un ambiente donde la manufactura esbelta se desarrolle con éxito. 
No se rechaza ninguna de las hipótesis de investigación, ya que los elementos individuales de estudio reflejan que el costo fue menor en el tercer trimestre de estudio del 2013.

Se concluye que el esfuerzo en conjunto de un equipo de trabajo puede llegar a ser lo más trascendente dentro de la implementación de iniciativas a nivel industrial ya que es ahí donde se capitalizan las ideas y logran tomar un impulso suficiente como para mover los resultados con solidez y crecimiento sostenido.

Los directivos y gerentes de las organizaciones que deseen implementar, desarrollar y sostener la filosofía de la mejora continua, deben comenzar con el tema del factor humano como paso primario del modelo de implementación que hayan elegido. En esta investigación se demuestra la jerarquía de la sensibilización y la necesidad de motivar al personal para involucrarlo exitosamente en la implementación de un sistema esbelto.

\section{References:}

1. Ackoff, Rusell L. (2004). Transforming the Systems Movement. EUA: Universidad de Pensilvania.

2. Berawi, M. A. (2004). Quality revolution: leading the innovation and competitive advantages. International Journal of Quality \& Reliability Management, 21(4), 425-438.

3. Chandan, R. C. (2008). Manufacturing Yogurt and Fermented Milks. Iowa US: Blackwell Publishing.

4. Evans, J.R.; Lindsey, W.M. (2013) Managing for quality and performance excellence. United States. Cengage Learning.

5. Gutiérrez, G. (2000). Justo a Tiempo y Calidad Total, Principios y Aplicaciones. 5 ed. Ediciones Castillo S. A. de C. V., Monterrey, Nuevo León, México.

6. Krajewski. L. J. (2000). Administración de operaciones: estrategia y análisis. Pearson Educación. México.

7. Lefcovich, M. L. (2009). Reducción de costos mediante la asignación de la productividad a los costos. El Cid Editor.

8. Liker, J., Meier D. (2008). El Talento Toyota. México: Mc Graw Hill.

9. Glistau, E., \& Machado, N. I. C. (2008). Design Of Experiments And Six Sigma Methods In Logistics. Advanced Logistic systems, 2(1), 4754.

10. Niño, A., Olave, C. (2004). Modelo de aplicación de herramientas de Manufactura Esbelta desde el desarrollo y mejoramiento de la calidad en el sistema de producción. Bogotá.

11. Page, J. (2004). Implementing Lean Manufacturing Techniques. United States of America: Hanser Gardner Publications. 
12. Robbins, S. (2005) Comportamiento Organizacional. México: Pearson.

13. Torres, C. (2004). Despliegue de Seis Sigma en una organización: claves para el éxito. Buenos Aires, Argentina.

14. Vaughn, R. C. (2007). Control de Calidad. México: Limusa.

15. Wood, N. (2004) Lean Thinking: What it is and what it isn't. Management Services, (48) 2, 8 